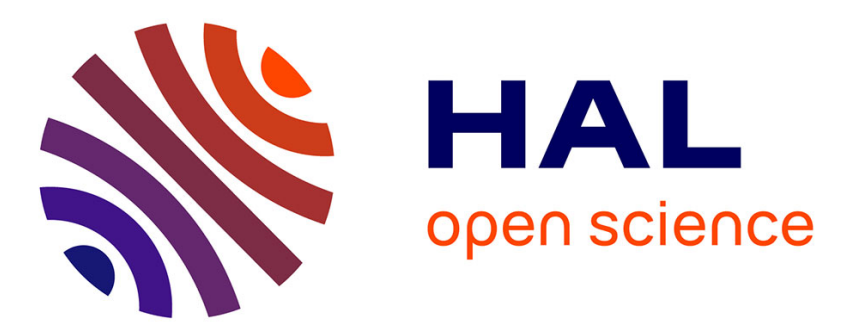

\title{
X-ray strain analysis of 111 fiber-textured thin films independent of grain-interaction models
}

Damien Faurie, Pierre Olivier Renault, Eric Le Bourhis, Thierry Chauveau, Olivier Castelnau, Philippe Goudeau

\section{- To cite this version:}

Damien Faurie, Pierre Olivier Renault, Eric Le Bourhis, Thierry Chauveau, Olivier Castelnau, et al.. $\mathrm{X}$-ray strain analysis of 111 fiber-textured thin films independent of grain-interaction models. Journal of Applied Crystallography, 2011, 44 (2), pp.409-413. 10.1107/S0021889811005115 . hal-02142286

\section{HAL Id: hal-02142286 \\ https://hal.science/hal-02142286}

Submitted on 28 May 2019

HAL is a multi-disciplinary open access archive for the deposit and dissemination of scientific research documents, whether they are published or not. The documents may come from teaching and research institutions in France or abroad, or from public or private research centers.
L'archive ouverte pluridisciplinaire HAL, est destinée au dépôt et à la diffusion de documents scientifiques de niveau recherche, publiés ou non, émanant des établissements d'enseignement et de recherche français ou étrangers, des laboratoires publics ou privés. 


\title{
$X$-ray strain analysis of $\{111\}$ fiber-textured thin films independent of grain-interaction models
}

\author{
D. Faurie, ${ }^{a} *$ P.-O. Renault, ${ }^{b}$ E. Le Bourhis, ${ }^{b}$ T. Chauveau, ${ }^{a}$ O. Castelnau ${ }^{c}$ and \\ Ph. Goudeau ${ }^{b}$
}

aLSPM, UPR 3407 CNRS, Université Paris-Nord, 93430 Villetaneuse, France, ${ }^{\mathbf{b}}$ Institut Pprime, CNRS - Université de Poitiers - ENSMA, UPR 3346, 86962 Futuroscope, France, and ${ }^{\text {}}$ PIMM, UMR 8006 CNRS, ENSAM Paris, 75013 Paris, France. Correspondence e-mail: faurie@univ-paris13.fr

\begin{abstract}
The anisotropic elastic response of supported thin films with a $\{111\}$ fiber texture has been studied using an in-situ micro-tensile tester and X-ray diffractometry. It is shown which specific X-ray diffraction measurement geometries can be used to analyze the elastic strains in thin films without requiring any assumptions regarding elastic interactions between grains. It is evidenced (theoretically and experimentally) that the combination of two specific geometries leads to a simple linear relationship between the measured strains and the geometrical variable $\sin ^{2} \psi$, avoiding the transition scale models. The linear fit of the experimental data allows a direct determination of the relationship between the three single-crystal elastic compliances or a direct determination of the $S_{44}$ single-crystal elastic compliance and the combination of $S_{11}+2 S_{12}$ if the macroscopic stress is known. This methodology has been applied to a model system, i.e. gold film for which no size effect is expected, deposited on polyimide substrate, and it was found that $S_{44}=23.2 \mathrm{TPa}^{-1}$ and $S_{11}+2 S_{12}=1.9 \mathrm{TPa}^{-1}$, in good accordance with values for large crystals of gold.
\end{abstract}

\section{Introduction}

For a few years, there has been an increasing interest in the elastic properties of thin films. Literature data show that the elastic behavior of metallic thin films differs significantly from that of their bulk counterparts because of their specific microstructure (texture, columnar grains, defects), their thickness and the grain-size (often nanometric) effect. Moreover, metallic thin films fabricated by physical vapor deposition usually exhibit strong crystallographic textures which induce macroscopic elastic anisotropy. Particularly, $\{111\}$ fiber texture is often encountered in the case of face-centered cubic (f.c.c.) materials such as gold. For elastically anisotropic solids, texture induces macroscopic elastic anisotropy whose amplitude depends both on the degree of texture and on the elastic anisotropy of the crystallites (local anisotropy) that compose the polycrystalline thin films (Matthies et al., 2001; Faurie et al., 2010). In many cases, the macroscopic elastic anisotropy is very high and cannot be neglected.

Several techniques are used to study elastic properties of polycrystalline thin films or multilayers, but most of these allow the determination of effective (macroscopic) elastic constants, such as, for example, acoustic spectroscopy (Nakamura et al., 2007; Tanei et al., 2008) and Brillouin light scattering (Martin et al., 2005) (few $\tilde{C}_{i j}$ ), tensile testing (Huang \& Spaepen, 2000; Wu et al., 2005) (Young's modulus $E$ and Poisson's ratio v) or nanoindentation (Cheng et al., 2006) (indentation modulus). However, determining single-crystal elastic constants $S_{i j}$ or $C_{i j}$ (local scale) from the thin-film effective elastic constants $\tilde{S}_{i j}$ or $\tilde{C}_{i j}$ (macroscopic scale) is cumbersome when local anisotropy is present. This requires at least a good knowledge of the microstructure and of the elastic coupling between crystallites in polycrystalline thin films. Actually, in our experience, the specific microstructure of thin films (small size and columnar grain structure) makes unsuitable the classical transition scale models such as those of Reuss (same stress in the grains) or Voigt (same strain in the grains) or the more sophisticated self-consistent model (Faurie, Castelnau et al., 2006; Faurie et al., 2009). On the other hand, the Vook-Witt model, which was expected to describe the true elastic behavior of thin films (Van Leeuwen et al., 1999), seems to be adapted only for ultra-thin films (a few tens of nanometres; Welzel et al., 2009). Although several grain interaction models have been proposed (Welzel et al., 2003; Baczmański et al., 2006, 2008; Liu et al., 2010), the actual geometrical arrangement of grains is still quite difficult to take into account.

In this paper, we show that X-ray strain analysis of $\{111\}$ fiber-textured films can be achieved without any assumptions regarding elastic grain interaction. This method can be used to obtain directly a combination of the three local elastic compliances or even to determine single-crystal elastic compliances $\left(S_{44}\right.$ and a combination of $S_{11}$ and $\left.S_{12}\right)$. It is based on tensile testing of thin films combined with X-ray diffraction (XRD) in specific geometries. Intragranular applied strains are determined from XRD peak shifts, while the overall 
stresses applied to the film-substrate composite are determined from a load cell incorporated into a micro-tensile tester. This is a general feature that is independent of the actual strain distribution within the diffracting volume, i.e. also valid for nanomaterials with a high volume fraction of grain boundaries exhibiting significant lattice disorder. We have applied this method to gold films on a polyimide substrate, a system extensively studied in previous work (Faurie et al., 2009, 2010).

\section{X-ray strain modeling}

\subsection{Theoretical background}

There are two contributions to the local elastic strain in the material. The first is due to the localization of the applied macroscopic stress in the different grains, associated with the purely elastic response of the specimen, which is the quantity of interest here. The second contribution is associated with the residual stress generated during the elaboration process. The advantage of performing in-situ experimental tests is that, once this second contribution has been characterized in the unloaded state, the purely elastic response of the specimen can be investigated, regardless of the residual stress level and distribution. In practice, this is achieved by measuring the shift of each Bragg peak with respect to its position in the unloaded configuration, which is a valid procedure as far as elasticity is concerned. The position of Bragg peaks (given by their center of gravity) provides a direct measurement of the average axial elastic strain (so-called 'lattice strain') in the diffracting volume and in a direction parallel to the scattering vector $\mathbf{q}$, given by the diffraction angles $\varphi$ and $\psi$ (see Fig. 1). The applied strain of an $h k l$ reflection is calculated using the unloaded state as a reference state:

$$
\{\varepsilon\}_{\varphi \psi}^{h k l}=\ln \left(\sin \theta_{\varphi \psi}^{T 0} / \sin \theta_{\varphi \psi}^{T 1}\right)
$$

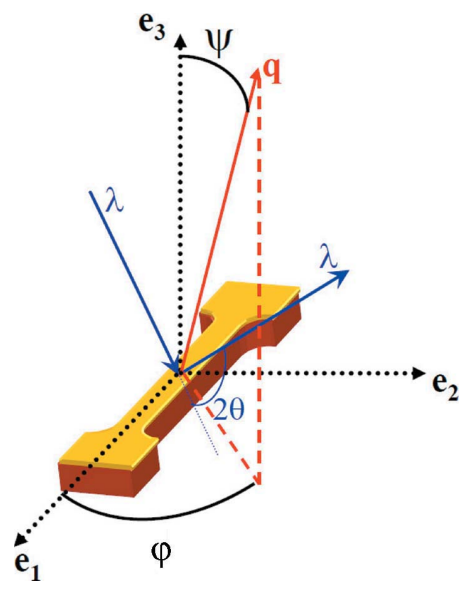

Figure 1

Geometry of the diffraction setup used for the in-situ loaded dogbone specimen. The uniaxial tensile stress applied to the film-substrate structure is along $\mathbf{e}_{1}$. The direction of the scattering vector $\mathbf{q}$ is defined by the in-plane azimuthal angle $\varphi$ from $\mathbf{e}_{1}$ and the polar angle $\psi$ from the sample normal direction $\mathbf{e}_{3} . \lambda$ is the wavelength of the X-rays. where $\theta_{\varphi \psi}^{T 0}$ is the angular position of the considered diffraction peak for the macroscopically unloaded state $T 0$ and $\theta_{\varphi \psi}^{T 1}$ the corresponding angles for the loaded state $T 1$. When applying a uniaxial test to a film-substrate composite, a biaxial stress state is applied in the metallic film. To interpret experimental data properly, it is necessary to estimate the overall stresses $\bar{\sigma}_{11}$ and $\bar{\sigma}_{22}$ to which the Au films are submitted. Recall that the specimens are made of a thick and compliant Kapton substrate, on top of which a thin and stiff Au layer has been deposited. The macroscopic stresses applied on the whole specimen by the tensile module localize differently in the two components, with a higher stress level in the $\mathrm{Au}$ layer. Following Castelnau et al. (2007), this effect can be calculated by replacing the polycrystalline $\mathrm{Au}$ layer with a uniform layer of equivalent stiffness $\tilde{C}$. This seems a reasonable assumption since the lateral dimension of the specimen (in-plane size) is much larger than the grain size. The transverse stress is induced by the mismatch of Poisson ratios between thin film and substrate. Obviously, the knowledge of $\bar{\sigma}_{11}$ and $\bar{\sigma}_{22}$ requires the estimation of macroscopic elastic constants, as extensively done in previous work (Faurie et al., 2009, 2010). For example, this problem could be simplified by studying selfsupported thin films for which the applied stress is known in a direct way.

In the case of a strongly textured thin film, the X-ray strain measurements can be performed for several $\{h k l\}$ plane families in the respective pole directions. The analytical solutions of X-ray strain for the Reuss and Voigt models for an ideal $\{111\}$ fiber texture have been derived for any $\varphi$ and $\psi$ values (Tanaka et al., 1999; Faurie, Renault et al., 2006; Yokoyama \& Harada, 2009). The analytical solution for the Reuss model is also known as the crystallite-group method (Welzel et al., 2005; Yokoyama et al., 2009). For a general nonequibiaxial stress state $\left(\bar{\sigma}_{11} \neq \bar{\sigma}_{22}\right)$, the Reuss model leads to the following equation:

$$
\begin{aligned}
\{\varepsilon\}_{\varphi \psi}^{h k l}= & \left(\bar{\sigma}_{11}-\bar{\sigma}_{22}\right)\left[\frac{S_{11}-S_{12}+S_{44}}{6} \cos (2 \varphi) \sin ^{2}\right. \\
& \left.+\left(-S_{11}+S_{12}+\frac{S_{44}}{2}\right) \frac{\sin (3 \beta+\varphi) \sin (2 \psi)}{3(2)^{1 / 2}}\right] \\
& +\left(\bar{\sigma}_{11}+\bar{\sigma}_{22}\right)\left(\frac{2 S_{11}+4 S_{12}-S_{44}}{6}+\frac{S_{44}}{4} \sin ^{2}\right) .
\end{aligned}
$$

The evolution of strain as a function of $\sin ^{2} \psi$ is not linear because of the term dependent on $\sin 2 \psi$. This nonlinearity increases with the local elastic anisotropy. However, it should be noted here that the nonlinearity is overestimated by the Reuss model (Faurie et al., 2009). Conversely, for a locally isotropic material (Zener elastic anisotropy factor $A=1$ ), $-S_{11}+S_{12}+S_{44} / 2=0$ and the term dependent on $\sin 2 \psi$ reduces to zero. The angle $\beta$ defines a rotation around the specimen surface normal direction. While the stress is applied at some fixed angle relative to the specimen axes, for each crystal and for particular diffraction geometries $\beta$ can take multiple values, corresponding to the crystallographic equivalent reflections. This orientation angle $\beta$ can be determined for each diffraction condition on the basis of a 
stereographic projection of poles for a cubic crystal with the $\{111\}$ pole at the center (for more details, see Yokoyama \& Harada, 2009). Moreover, the angle $\beta$ for $\varphi=0^{\circ}$ has to be replaced by $\beta-90^{\circ}$ for $\varphi=90^{\circ}$.

The Voigt model leads to the following equation (Tanaka et al., 1999; Faurie, Renault et al., 2006):

$$
\begin{aligned}
\{\varepsilon\}_{\varphi \psi}^{h k l}= & \left(\bar{\sigma}_{11}-\bar{\sigma}_{22}\right)\left[\frac{3\left(S_{11}-S_{12}\right) S_{44}}{2\left(4 S_{11}-4 S_{12}+S_{44}\right)} \cos (2 \varphi) \sin ^{2}\right] \\
& +\left(\bar{\sigma}_{11}+\bar{\sigma}_{22}\right)\left(\frac{2 S_{11}+4 S_{12}-S_{44}}{6}+\frac{S_{44}}{4} \sin ^{2}\right) .
\end{aligned}
$$

For the Voigt model the diffraction strain varies linearly with $\sin ^{2} \psi$, regardless of the local anisotropy of the material. This behavior directly results from the assumptions made in this model, i.e. equal strain for all the crystallites that compose the polycrystal. It should be noted here that the X-ray strain is $\varphi$ dependent for the two extreme models, resulting from the non-equibiaxial stress state $\left(\bar{\sigma}_{11} \neq \bar{\sigma}_{22}\right)$.

\subsection{Proposed approach}

A notable way to analyze X-ray strains in the case of $\{111\}$ fiber texture is to calculate the average between strains measured in the longitudinal $\left(\varphi=0^{\circ}\right)$ and transverse $\left(\varphi=90^{\circ}\right)$ directions, i.e.

$$
\{\varepsilon\}_{\text {average }}=\frac{\{\varepsilon\}_{0^{\circ},}^{h k l}+\{\varepsilon\}_{90^{\circ}}^{h k l}}{2}
$$

This average leads to a single linear strain- $\sin ^{2} \psi$ relationship, which is the same for the Reuss and Voigt models:

$$
\left.\{\varepsilon\}_{\text {average }}=\left(\bar{\sigma}_{11}+\bar{\sigma}_{22}\right) \frac{2 S_{11}+4 S_{12}-S_{44}}{6}+\frac{S_{44}}{4} \sin ^{2}\right) .
$$

The expression of $\{\varepsilon\}_{\text {average }}$ can also be derived in terms of effective stiffnesses $\tilde{C}_{i j}$ :

$$
\{\varepsilon\}_{\text {average }}=\left(\frac{\bar{\sigma}_{11}+\bar{\sigma}_{22}}{M \tilde{C}_{33}}\right)\left[-2 \tilde{C}_{13}+\left(2 \tilde{C}_{13}+\tilde{C}_{33}\right) \sin ^{2}\right],
$$

where $M=\tilde{C}_{11}+\tilde{C}_{12}-2 \tilde{C}_{13}^{2} / \tilde{C}_{33}$ is the so-called biaxial modulus that is independent of the adopted micromechanical model (Huang \& Weaver, 2005) for a $\{111\}$ fiber texture. This is the case also for $\tilde{C}_{13}$ and $\tilde{C}_{33}$ (Kröner \& Wawra, 1978). Obviously, any bounds that are better than those of Voigt and Reuss must also coincide if the Reuss and Voigt models coincide (Kröner \& Wawra, 1978). These effective elastic constants can be written as a function of single-crystal elastic compliances as follows:

$$
\begin{gathered}
\tilde{C}_{13}=\frac{-2 S_{11}-4 S_{12}+S_{44}}{3\left(S_{11}+2 S_{12}\right) S_{44}}, \\
\tilde{C}_{33}=\frac{4 S_{11}+8 S_{12}+S_{44}}{3\left(S_{11}+2 S_{12}\right) S_{44}},
\end{gathered}
$$

$$
M=\frac{6}{4 S_{11}+8 S_{12}+S_{44}} .
$$

Hence, the proposed method allows a linear fit of equation (5) to the experimental $\{\varepsilon\}_{\text {average }}$, regardless of the grain-interaction model and of the local elastic anisotropy level. Obviously, this method is only valid for sharp $\{111\}$ fiber textures, with a texture dispersion not higher than $10^{\circ}$ at half maximum (Faurie et al., 2010), as studied here.

\section{Thin-film fabrication and experimental setup}

We performed a tensile test of a locally elastically anisotropic gold film, deposited on a $127.5 \mu$ m-thick polyimide (Kapton) substrate, with in-situ X-ray strain analysis. A $700 \mathrm{~nm}$-thick gold film was deposited by physical vapor deposition (cathodic sputtering) and showed a strong $\{111\}$ fiber texture commonly encountered for f.c.c. materials (see pole figures in Fig. 2). $\mathrm{X}$-ray pole figure experiments were performed using a fourcircle diffractometer in Bragg-Brentano geometry, and X-rays (cobalt line focus source at $\lambda=0.179 \mathrm{~nm}$ ) were emitted by a Bruker rotating anode.

The average columnar grain diameter was estimated by transmission electronic microscopy (not shown here) to be $\sim 100 \mathrm{~nm}$, i.e. large enough for size effects on the single-crystal
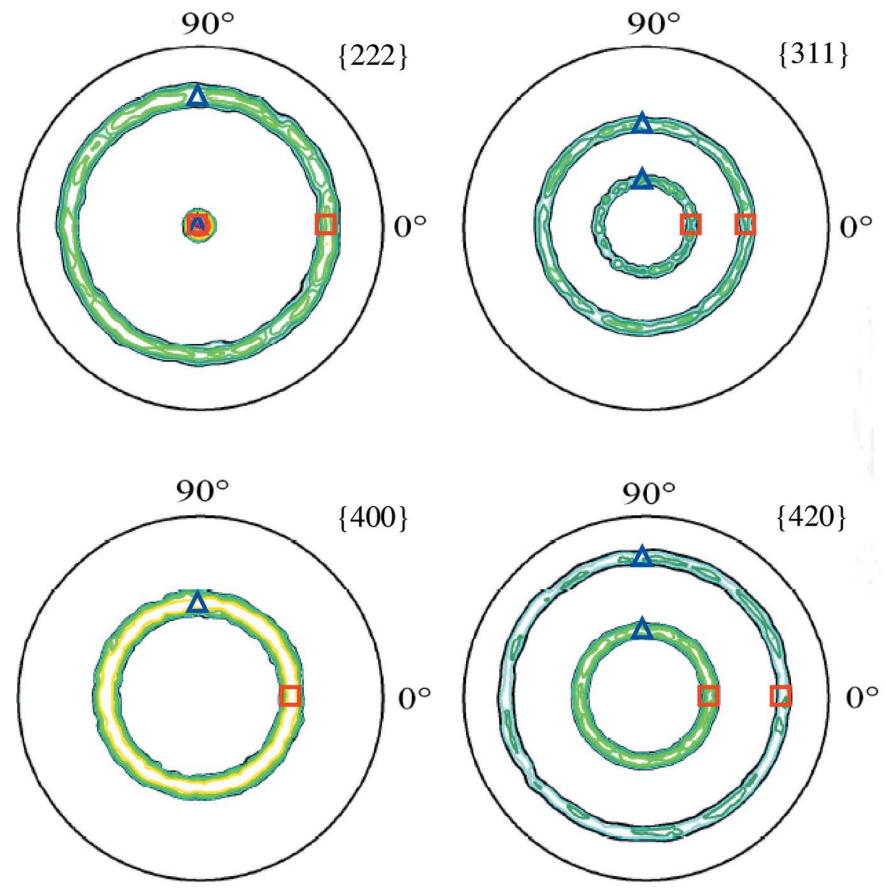

$$
\begin{array}{ll|l|l|l|l|l|l|l|l|l}
\begin{array}{l}
\text { Intensity } \\
\text { levels }
\end{array} & & & & & & & & & & \\
\end{array}
$$

Figure 2

X-ray pole figures for the $\{222\},\{311\},\{400\}$ and $\{420\}$ plane families. A measured $\{331\}$ pole figure is not shown here. The central peak of the $\{222\}$ pole figure and the measured rings are typical of a $\{111\}$ fiber texture. The FWHM of the central peak is about $8^{\circ}$. The red square and blue triangle symbols represent the pole directions at $\varphi=0^{\circ}$ and $\varphi=90^{\circ}$, respectively. 
elastic constants to be neglected at room temperature (Schiotz et al., 1999). The single-crystal elastic constants of gold, known for large crystals, are $S_{11}=23.3 \mathrm{TPa}^{-1}, S_{12}=-10.7 \mathrm{TPa}^{-1}$ and $S_{44}=23.8 \mathrm{TPa}^{-1}$ (Smithells, 1976). The corresponding Zener elastic anisotropy factor $A=2\left(S_{11}-S_{12}\right) / S_{44}$ is relatively high ( $A \simeq 2.8$ ), indicating a quite large elastic anisotropy at the grain scale.

The in-situ X-ray diffraction experiments were performed at the LURE synchrotron radiation facility on beamline DW22 (CNRS, Orsay, France). The wavelength of the beam $(\lambda=$ $0.161 \mathrm{~nm}$ with a spectral resolution of $\Delta q / q=5.10^{-4}$ ) was selected by a double-bounce $\operatorname{Si}(111)$ monochromator. The beam size was set to $100 \times 500 \mu \mathrm{m}$, that is, much larger than the average in-plane grain size of the gold films, and the vertical beam divergence can be evaluated to be less than $0.006^{\circ}$. The tensile module was at the center of a four-circle diffractometer (with an angular resolution of $0.001^{\circ}$ for the four angles) so that X-ray diffraction could be carried out on loaded specimens. The external load was applied to the composite (film and substrate) dogbone-shaped specimens by means of a $200 \mathrm{~N}$ Deben tensile module equipped with a $20 \mathrm{~N}$ load cell enabling force measurement with a precision better than $0.1 \mathrm{~N}$. With our experimental conditions, the uniaxial load $T 1(F=6.5 \mathrm{~N})$ applied to the film/substrate structure induces longitudinal and transverse macroscopic stresses in the films, determined to be $\bar{\sigma}_{11}=170 \mathrm{MPa}$ and $\bar{\sigma}_{22}=36 \mathrm{MPa}$, respectively. In the present experiment, we applied the load increment and then waited for about $600 \mathrm{~s}$ before starting the $\mathrm{X}$-ray measurements. In this case, the relaxation of the polyimide can be neglected: it only contributes about $3 \%$ of the applied load increment.

Because of the pronounced crystallographic texture, measurements were performed only for specific orientations of $\mathbf{q}$, those for which the intensity is high enough (pole directions). The corresponding $\psi$ values for each $\{h k l\}$ plane

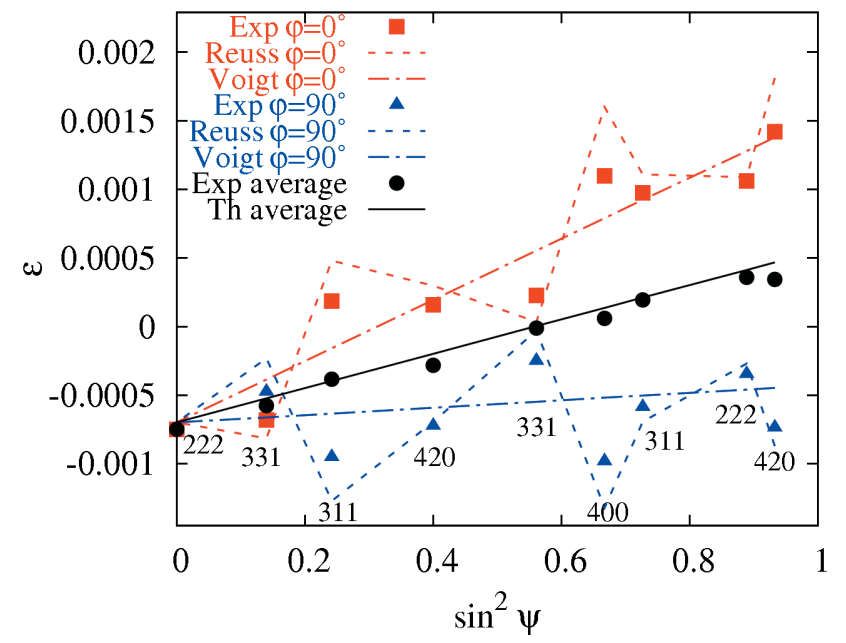

Figure 3

X-ray strains $\varepsilon$ as a function of $\sin ^{2} \psi$ for the Reuss and Voigt models (lines), and experimental results (red square symbols for $\varphi=0^{\circ}$ and blue triangle symbols for $\varphi=90^{\circ}$ ). The theoretical and experimental averages are represented by a continuous black line and black circles, respectively. Here, the strains were calculated using large crystal compliances, $S_{11}=$ $23.3 \mathrm{TPa}^{-1}, S_{12}=-10.7 \mathrm{TPa}^{-1}$ and $S_{44}=23.8 \mathrm{TPa}^{-1}$. family are given by Faurie et al. (2009). As proposed in $\$ 2.2$, the measurements were performed for two $\varphi$ values ( 0 and $\left.90^{\circ}\right)$.

\section{Experimental results and discussion}

In Fig. 3, we show the experimental and theoretical results for the two studied geometries [longitudinal $\left(\varphi=0^{\circ}\right)$ and transverse $\left.\left(\varphi=90^{\circ}\right)\right]$, using the single-crystal compliances known for large crystals, since size effects can be neglected here. Experimental lattice strains are plotted against $\sin ^{2} \psi$, and complex variations are obtained for both $\varphi$ values $\left(0\right.$ and $\left.90^{\circ}\right)$. The strongly nonlinear character of the plot already indicates that single-crystal elastic anisotropy plays an important role. Moreover, this experimental behavior is not captured by the two extreme models: the Voigt bound predicts a linear behavior, while the nonlinearity is overestimated by the Reuss bound. In contrast, the experimental arithmetic average follows a linear behavior and is very well captured by the theoretical arithmetic average $\{\varepsilon\}_{\text {average }}$, which is independent of the grain-interaction models. From equation (5), it is clearly seen that a relationship among elastic compliances can be directly extracted, i.e. $2\left(2 S_{11}+4 S_{12}-S_{44}\right) /\left(-3 S_{44}\right)$. Indeed, this ratio is simply deduced from the slope and the origin ordinate of the experimental linear fit. In this study, we find 0.563 , which is close to the value obtained for large crystals of gold (0.560). It should be noted that this value can be determined graphically, since it corresponds to the $\sin ^{2} \psi$ value for $\{\varepsilon\}_{\text {average }}=0$. Thus some changes in local elastic constants of thin films could be directly captured without any assumptions regarding the grain-interaction model. Such experiments may be useful in the case of nanocrystalline materials (grain size smaller than 5-10 $\mathrm{nm}$ ), for which drastic changes are expected relative to their coarse-grain counterparts (Latapie \& Farkas, 2003; Zhao et al., 2006). Moreover, assuming that the stresses applied to the film-substrate composite are known, a reciprocal approach variation can be used, namely adjusting $\{\varepsilon\}_{\text {average }}$ linearly to extract local elastic compliances $\left(S_{44}\right.$ and the combination $S_{11}+2 S_{12}$ ). Here the best adjustment leads to $S_{44}=23.2 \mathrm{TPa}^{-1}$ and $S_{11}+2 S_{12}=1.8 \mathrm{TPa}^{-1}$, which are close to the values for large crystals $\left(S_{44}=23.8 \mathrm{TPa}^{-1}\right.$ and $S_{11}+2 S_{12}=1.9 \mathrm{TPa}^{-1}$ ).

It should be noted here that this method could be applied to 'as-deposited' $\{111\}$ fiber-textured thin films if the residual stress is known, for example, by a sample curvature measurement method, as already developed by Kamminga (1999) and Martinschitz et al. (2009) for extracting, respectively, Young's moduli and $h \mathrm{kl}$-dependent Young's moduli of thin films on rigid substrates. The residual stress state is generally equibiaxial $\left(\bar{\sigma}_{11}=\bar{\sigma}_{22}\right)$, leading to the same $\varphi$-independent simple expression of the X-ray strain in equations (2) and (3).

\section{Conclusion}

In summary, the proposed approach combining two experimental configurations [measurements performed for two $\varphi$ 
values $\left(0\right.$ and $\left.\left.90^{\circ}\right)\right]$ allows $X$-ray strain analysis of $\{111\}$ fibertextured films regardless of grain-interaction models. In fact, the average strain $\{\varepsilon\}_{\text {average }}$ (that is, the average of the two $\mathrm{X}$-ray strains obtained in the proposed configurations, namely longitudinal and transverse) is shown to vary linearly with $\sin ^{2} \psi$, the involved features being independent of the graininteraction model. Moreover, this analysis can be used to extract elastic constants of elastically anisotropic films, this method being available for any in-plane biaxial stress state.

The authors wish to thank M. Drouet for thin-film deposition, and E. Elkaïm and S. Rouzière for their help in conducting the synchrotron experiments. This work has been supported by the French Agency for Research (ANR) through the Pnano project entitled Cmonano (ANR-05-PNANO-069).

\section{References}

Baczmanski, A., Lipinski, P., Tidu, A., Wierzbanowski, K. \& Pathiraj, B. (2008). J. Appl. Cryst. 41, 854-867.

Baczmański, A., Tidu, A., Lipinski, P., Humbert, M. \& Wierzbanowski, K. (2006). Mater. Sci. Forum, 524-525, 235-240.

Castelnau, O., Geandier, G., Renault, P.-O., Goudeau, Ph. \& Le Bourhis, E. (2007). Thin Solid Films, 516, 320-324.

Cheng, Y. T., Ni, W. \& Cheng, C. M. (2006). Phys. Rev. Lett. 97, 075506.

Faurie, D., Castelnau, O., Brenner, R., Renault, P.-O., Le Bourhis, E. \& Goudeau, Ph. (2009). J. Appl. Cryst. 42, 1073-1084.

Faurie, D., Castelnau, O., Renault, P.-O., Brenner, R., Le Bourhis, E., Goudeau, Ph. \& Patriarche, G. (2006). Appl. Phys. Lett. 89, 061911.

Faurie, D., Djemia, P., Le Bourhis, E., Renault, P.-O., Roussigné, Y., Chérif, S. M., Brenner, R., Castelnau, O., Patriarche, G. \& Goudeau, Ph. (2010). Acta Mater. 58, 4998-5008.
Faurie, D., Renault, P.-O., Le Bourhis, E. \& Goudeau, Ph. (2006). Acta Mater. 54, 4503-4513.

Huang, F. \& Weaver, M. L. (2005). J. Appl. Phys. 98, 073505.

Huang, H. \& Spaepen, F. (2000). Acta Mater. 48, 3261-3269.

Kamminga, J. D. (1999). PhD thesis, Delft University of Technology, The Netherlands.

Kröner, E. \& Wawra, H. H. (1978). Philos. Mag. A, 38, 433-437.

Latapie, A. \& Farkas, D. (2003). Scr. Mater. 48, 611-615.

Liu, C. Q., Li, W. L. \& Fei, W. D. (2010). Acta Mater. 58, 5393-5401.

Martin, F., Jaouen, C., Pacaud, J., Abadias, G., Djemia, Ph. \& Ganot, F. (2005). Phys. Rev. B, 71, 045422.

Martinschitz, K. J., Daniel, R., Mitterer, C. \& Keckes, J. (2009). J. Appl. Cryst. 42, 416-428.

Matthies, S., Priesmeyer, H. G. \& Daymond, M. R. (2001). J. Appl. Cryst. 34, 585-601.

Nakamura, N., Ogi, H. \& Hirao, M. (2007). Phys. Rev. B, 77, 245416.

Schiotz, J., Vegge, T., Di Tolla, F. D. \& Jacobsen, K. W. (1999). Phys. Rev. B, 60, 11971-11983.

Smithells, J. C. (1976). Metals Reference Book. London: Butterworths. Tanaka, K., Akiniwa, Y., Ito, T. \& Inoue, K. (1999). Jpn Soc. Mech. Eng. Int. J. Ser. A, 42, 224-234.

Tanei, H., Nakamura, N., Ogi, H., Hirao, M. \& Ikeda, R. (2008). Phys. Rev. Lett. 100, 016804.

Van Leeuwen, M., Kamminga, J.-D. \& Mittemeijer, E. J. (1999). J. Appl. Phys. 86, 1904-1914.

Welzel, U., Kumar, A. \& Mittemeijer, E. J. (2009). Appl. Phys. Lett. 95, 111907.

Welzel, U., Leoni, M. \& Mittemeijer, E. J. (2003). Philos. Mag. 83, 603-630.

Welzel, U., Ligot, J., Lamparter, P., Vermeulen, A. C. \& Mittemeijer, E. J. (2005). J. Appl. Cryst. 38, 1-29.

Wu, B., Heidelberg, A. \& Boland, J. J. (2005). Nat. Mater. 4, 525-529.

Yokoyama, R. \& Harada, J. (2009). J. Appl. Cryst. 42, 185-191.

Yokoyama, R., Harada, J. \& Akiniwa, Y. (2009). J. Appl. Cryst. 42, 776-782.

Zhao, S.-J., Albe, K. \& Hahn, H. (2006). Scr. Mater. 55, 473-476. 